\section{Actively targeting mTOR}

Many tumour cells carry mutations that increase signalling through the PI3K-AKT-mTOR (phosphoinositide 3-kinase-AKT-mammalian target of rapamycin) pathway. Although rapamycin (also known as sirolimus), which suppresses the kinase activity of mTOR through an allosteric mechanism, is used in cancer therapy it does not fully inhibit either of the protein complexes - TORC1 (target of rapamycin complex 1) and TORC2 - that contain mTOR.

In an effort to overcome this limitation, four independent groups recently identified selective inhibitors that target the ATP catalytic binding site of the mTOR kinase, sometimes known as the active site. In their study, reported in Nature Medicine, Fruman and colleagues compared rapamycin and one TORC1 and TORC2 inhibitor, PP242, in models of leukaemia.

In cellular models of B precursor acute lymphoblastic leukaemia (B-ALL) positive for the Philadelphia chromosome $\left(\mathrm{Ph}^{+}\right)$- which results in the production of the deregulated kinase BCR-ABL - PP242 suppressed cell growth by $>90 \%$, with low nanomolar potency, whereas rapamycin inhibited only $~ 60 \%$ of cell growth. PP242 also had greater antiproliferative potency relative to rapamycin in a number of solid tumour cell lines that carried either PI3K gain-of-function mutations or PTEN (phosphatase and tensin homologue) loss-of-function mutations. This suggests that inhibitors of TORC1 and TORC2 have a stronger anticancer effect.
PP242 reduced colony formation in primary cells from patients with newly diagnosed $\mathrm{Ph}^{+} \mathrm{B}$-ALL. It also augmented the effect of the secondgeneration $\mathrm{BCR}-\mathrm{ABL}$ inhibitor dasatinib (Sprycel; Bristol-Myers Squibb) in samples derived from subjects who had relapsed after combination chemotherapy with the pioneering BCR-ABL inhibitor imatinib (Glivec/ Gleevec; Novartis). Further studies revealed that PP242 and rapamycin had distinct effects on phosphoprotein levels mediated by TORC1 and TORC2 signalling; PP242 blocked all TORC1 and TORC2 outputs without modulating the activity of PI3K.

To compare PP242 with rapamycin in vivo, the authors used three mouse leukaemia models. In a syngeneic $B C R-A B L$ pre- $B$ lymphoid model, short-term oral dosing with PP242 reduced leukaemic burden in the spleen and bone marrow, whereas rapamycin did not. In a long-term survival study, oral dosing of PP242 combined with imatinib provided the greatest survival benefit. A xenograft model of human $\mathrm{Ph}^{+} \mathrm{B}$-ALL showed that the combination of dasatinib (which is emerging as the standard of care for $\mathrm{Ph}^{+} \mathrm{B}-\mathrm{ALL}$ ) with PP242 caused regression of leukaemia and prevented dissemination to the central nervous system. Although rapamycin enhanced the effect of dasatinib, leukaemic burden continued to increase with this combination. In xenograft experiments using primary human leukaemia samples, PP242 in combination with dasatinib suppressed the expansion of some $\mathrm{Ph}^{+} \mathrm{B}$-ALL samples, but not others.

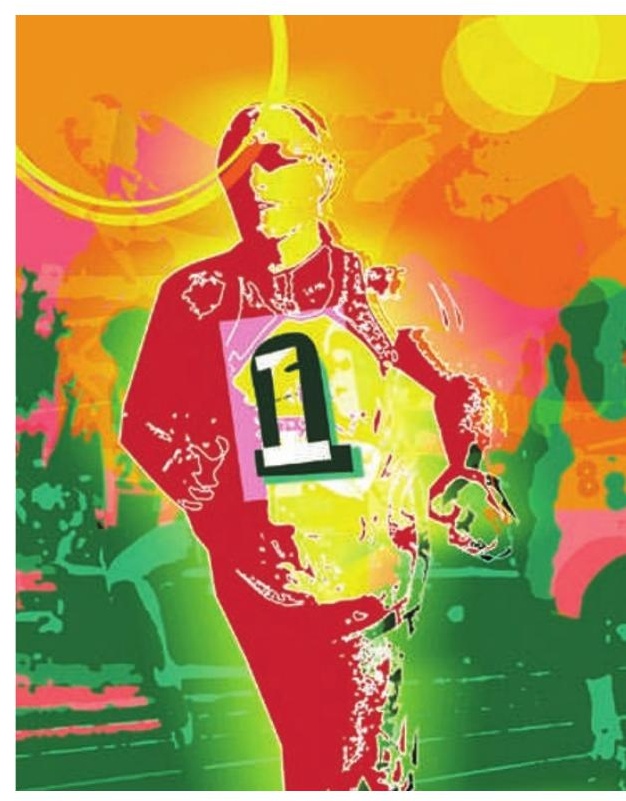

Importantly, PP242 showed consistently weaker effects than rapamycin in assays of adaptive immune function, indicating that it was less immunosuppressive than rapamycin. The compound also had a more favourable balance of efficacy and tolerability than PI-103, an inhibitor of PI3K, and TORC1 and TOR2. So, selective inhibitors of TORC1 and TORC2 might be superior to those that also inhibit PI3K (several of which are in clinical trials) and to rapamycin, and might be particularly effective when used with regimens that include dasatinib for treating $\mathrm{Ph}^{+} \mathrm{B}-\mathrm{ALL}$.

Charlotte Harrison

ORIGINAL RESEARCH PAPER Janes, M. R. et al. Effective and selective targeting of leukemia cells using a TORC $1 / 2$ kinase inhibitor. Nature Med. 16, 205-213 (2010) 